\title{
Relações fisiológicas em mudas de pata-de-vaca (Bauhinia forficata Link)
}

CARON, B.O..$^{*}$; PERRANDO, E.R. ${ }^{2}$; SCHMIDT, D. ${ }^{1}$; MANFRON, P.A. ${ }^{1}$; BEHLING, A. ${ }^{3}$; ELLI, E.F. ${ }^{1}$; ELOY, E. ${ }^{3}$ ${ }^{1}$ Departamento de Ciências Agronômicas e Ambientais, Centro de Educação Superior Norte do Rio Grande do Sul (CESNORS), Universidade Federal de Santa Maria (UFSM), Linha Sete de Setembro s/n, BR386 Km40, CEP98400-000, Frederico Westphalen (RS), Brasil. *otomarcaron@yahoo.com.br. ${ }^{2}$ Departamento de Engenharia Florestal, Centro de Educação Superior Norte do Rio Grande do Sul (CESNORS), Universidade Federal de Santa Maria (UFSM), Linha Sete de Setembro s/n, BR386 Km 40, CEP 98400-000, Frederico Westphalen (RS), Brasil. ${ }^{3}$ Departamento de Engenharia Florestal, Universidade Federal do Paraná (UFPR), Av. Pref. Lothário Meissner, 900, Jardim Botânico, Campus III, CEP 80210-170, Curitiba (PR), Brasil.

\begin{abstract}
RESUMO: Os fatores ambientais, tais como: temperatura do ar, umidade relativa do ar e radiação solar, influenciam no comportamento fisiológico dos vegetais refletindo no seu crescimento e desenvolvimento. O objetivo do trabalho foi avaliar o comportamento fisiológico em mudas de pata-de-vaca ao longo do dia e constatar qual o melhor horário para a realização de determinações de trocas gasosas. Para tanto, foram realizadas avaliações de temperatura da folha, radiação fotossinteticamente ativa, resistência estomática e transpiração, utilizando-se um porômetro, das 8:00 às 17:00 horas, em intervalos de uma hora entre as medidas. A espécie apresenta oscilações no seu mecanismo fisiológico em função do horário de avaliação e das condições climáticas, sendo o intervalo mais indicado para a realização de determinações das trocas gasosas das 10:00 às 13:00 horas.
\end{abstract}

Palavras-chave: Estresse hídrico, elementos ambientais, resistência estomática.

\begin{abstract}
Physiologic relationships in Brazilian Orchid Tree (Bauhinia forficata Link) seedlings. Environmental elements, such as air temperature, relative humidity and solar radiation, affect the physiological behavior of plants, which is reflected in their growth and development. The objective of this study was to evaluate the physiological changes of Brazilian Orchid Tree seedlings over a typical day and find the best time to carry out determinations of gas exchanges. For this end, we evaluated temperature of the leaf, photosynthetically active radiation, stomatal resistance and perspiration, using a porometer, from 8:00 a.m. to 05:00 p.m., in intervals of one hour between measurements. The species presents oscillations in its physiologic mechanism in function of the time of evaluation and climatic conditions, being the most suitable interval to determine gas exchanges from 10:00 a.m. to 01:00 p.m.
\end{abstract}

Keywords: Water stress, environmental elements, stomatal resistance.

\section{INTRODUÇÃO}

A espécie Bauhinia forficata Link, popularmente conhecida como pata-de-vaca, (Agra et al., 2007; Menezes et al., 2007) vem conquistando o interesse de vários pesquisadores, pelo fato de estudos fitoquímicos comprovarem a identificação de um marcador químico localizado nas folhas chamado "kaempferitrina" e que confere a espécie a atividade hipoglicemiante (Silva et al., 2000; Silva \& Cechinel-Filho, 2002, Sousa et al., 2004), além de apresentar funções diuréticas e ser utilizada para combater inflamações renais, o que lhe confere a classificação de planta medicinal, sendo seu uso conhecido popularmente (Lorenzi, 2002).

A pata-de-vaca é nativa do Sul do Brasil, Paraguai, Argentina e Uruguai, se desenvolve principalmente no entorno das matas, pode ser encontrada sob forma de arbusto ou árvore com aproximadamente seis metros de estatura. As plantas, além de possuírem propriedades medicinais, se destacam por apresentarem características paisagísticas adequadas para arborização urbana, tais como: porte médio, folhas grandes, largura de copa mediana, e flores com admirável aspecto visual (Rosa et al., 2008). 
A sazonalidade dos elementos climáticos como temperatura do ar, umidade relativa do ar e radiação solar podem alterar o comportamento fisiológico dos vegetais e, consequentemente o crescimento e desenvolvimento dos mesmos, uma vez que, cerca de $90 \%$ da produção biológica das plantas ocorre em resposta à atividade fotossintética e o aumento na resistência difusiva estomática pode ocasionar diminuição na fotossíntese líquida (Floss, 2004; Amaral et al., 2006). Logo, por ser o componente kaempferitrina um metabólico secundário, a variação dos elementos climáticos pode afetar seus valores, visto que, muitos deles são voláteis, podendo-se perder componentes importantes no rendimento final do mesmo (Suarez et al., 2003).

O conteúdo de água no solo e nos tecidos das plantas, a temperatura do ar, a intensidade luminosa, a concentração de gás carbônico $\left(\mathrm{CO}_{2}\right)$ no ar, e o teor de nitrogênio das folhas das plantas, são também fatores que afetam a atividade fotossintética dos vegetais (Marenco \& Lopes, 2005). O processo de abertura e fechamento dos estômatos está relacionado com a intensidade de luz e o teor de água contido nas folhas das plantas; logo, a frequência da funcionalidade dos estômatos e a área foliar influenciam diretamente na produtividade dos vegetais (Costa \& Marenco, 2007).

Neste contexto, a verificação das trocas gasosas constitui uma importante ferramenta a fim de diagnosticar a adaptabilidade e estabilidade de plantas a determinados ecossistemas, uma vez que a redução do crescimento das plantas pode estar relacionada à redução na atividade fotossintética, limitada por fatores abióticos intrínsecos ao local de cultivo (Peixoto et al., 2002, Paiva et al., 2005).

Outro ponto a ser considerado é que nos diferentes horários do dia, pode haver comportamentos fisiológicos distintos na planta. Para Jones et al. (1982) em horas próximas ao meio dia, as alterações da taxa transpiratória e do potencial de água na folha são proporcionalmente reduzidas, permitindo relativo equilíbrio dinâmico do sistema solo-planta-atmosfera. Assim, justifica-se a grande importância em se determinar o horário adequado para a realização das trocas gasosas.

Os estudos fisiológicos para obtenção de informações sobre as plantas classificadas como medicinais são pouco realizados e difundidos no meio científico, ainda que tais fatores venham afetar o crescimento, o desenvolvimento, e a produção de diferentes metabólicos (Taiz \& Zeiger, 2009).

Frente ao exposto, o objetivo do trabalho foi avaliar o comportamento estomático em mudas de pata-de-vaca ao longo de um dia típico, e constatar qual o melhor horário para a realização de determinações das trocas gasosas.

\section{MATERIAL E MÉTODO}

O experimento foi conduzido em casa de vegetação, no viveiro florestal do Centro de Educação Superior Norte do Rio Grande do Sul (CESNORS/UFSM), localizado no Campus de Frederico Westphalen - RS, situado na BR 386, linha Sete de Setembro, região do Médio-Alto Uruguai, cujas coordenadas geográficas são: $27^{\circ} 23^{\prime} 26^{\prime \prime S}$; $53^{\circ} 25^{\prime} 43^{\prime \prime} \mathrm{W}$ e 641 metros de altitude.

Segundo a classificação climática de Köppen, o clima da região é Cfa. Frederico Westphalen está distante de Iraí aproximadamente $30 \mathrm{~km}$, sendo o município tomado como referência para os dados de classificação climática. Conforme proposta de MALUF (2000), Iraí apresenta clima de tipo subtemperado subúmido, sendo a temperatura média anual de $18,8^{\circ} \mathrm{C}$ e temperatura média do mês mais frio de $13,3^{\circ} \mathrm{C}$.

Foi utilizado o delineamento experimental inteiramente casualizado, com três repetições, sendo avaliadas três plantas para cada repetição, totalizando nove mudas. Como suporte para os tubetes foram utilizadas bandejas de polietileno com capacidade para 54 mudas, as quais foram dispostas a 1,0 m do nível do solo.

Para superação da dormência das sementes utilizou-se imersão em água quente $\left(80^{\circ} \mathrm{C}\right)$ e permanência na água fora do aquecimento por 10 minutos para embebição das sementes (Carvalho, 1994). Durante a condução do experimento o regime hídrico na casa de vegetação foi sistematizado em três irrigações diárias através de aspersores a fim de manter a capacidade de pote (CP). A CP foi adotada como sendo o conteúdo de água retida pelo solo após sofrer saturação e consequente ação da gravidade, até o cessamento da drenagem (SOUZA et al., 2000).

Asemeadura foi realizada no dia 15/09/2010, em tubetes de polietileno $(13,2 \mathrm{~cm}$ de altura $\times 5,2 \mathrm{~cm}$ de diâmetro), preenchidos com substrato comercial $\left(\right.$ PLANTMAX $\left.^{\circledR}\right)$ e fertilizante de liberação lenta $\left(\right.$ Basacote $^{\circledR}$ ) na dose de $6 \mathrm{~kg} \mathrm{~m}^{-3}$ de substrato.

As características avaliadas foram: temperatura da folha $\left({ }^{\circ} \mathrm{C}\right)$, radiação fotossinteticamente ativa $\left(\mu \mathrm{mol} \mathrm{S}{ }^{-1} \mathrm{~m}^{-2}\right)$, resistência estomática $\left(\mathrm{S} \mathrm{cm}^{-1}\right)$ e transpiração $\left(\mathrm{mmol} \mathrm{H}_{2} \mathrm{O} \mathrm{s}^{-1}\right.$ $\mathrm{m}^{-2}$ ) ao longo de um dia, com uso de um porômetro digital LI-1600 LI-COR. As medidas da temperatura e umidade relativa do ar foram realizadas fora e dentro da estufa, utilizando-se um termohigrógrafo.

As avaliações foram realizadas no dia 09/03/2011, aos 155 dias após a emergência, considerado dia típico em agroclimatologia, entre 8:00 e 17:00h, com intervalo de 60 minutos. Este dia foi escolhido, entre tantos da estação de verão, por apresentar a abóboda celeste em condições de nebulosidade zero, permitindo assim, a obtenção 
dos máximos efeitos dos elementos climáticos sobre o ambiente de cultivo e, consequentemente, sobre as características analisadas conforme os objetivos do trabalho.

Os dados foram submetidos à análise estatística através do Software "Statistical Analysis System" (SAS, 2003), sendo realizada a correlação de Pearson, classificada quanto ao grau de dependência proposto por Steel \& Torrie (1980) como: muito fraca $(0-0,3)$, fraca $(0,3-0,5)$, moderada $(0,5-0,7)$, alta $(0,7-0,9)$ e altíssima (maior que 0,9 ).

\section{RESULTADO E DISCUSSÃO}

A máxima radiação fotossinteticamente ativa (RFA) ocorreu às 12:00h com o valor de 972,189 umol $\mathrm{S}^{-1} \mathrm{~m}^{-2}$ e a máxima temperatura foliar foi identificada às $13: 00 \mathrm{~h}$ no valor de $30,4^{\circ} \mathrm{C}$ (Figura 1 ). Estas variáveis apresentaram correlação moderada entre si $(0,66)$ devido à temperatura da estufa influenciar fortemente na variação destes valores, e, consequentemente, resultar em altas magnitudes de correlação (RFA de 0,87; Temperatura foliar de 0,91), de acordo com a Tabela 1.

Valores semelhantes foram encontrados por Jadoski et al. (2005) analisando plantas de Capsicum annuum L. ao longo do dia 13/03/2002, em estufa tipo túnel alto, onde verificaram a máxima RFA entre 11:30h e 13:30h e as temperaturas máximas entre 13:30h e 15:30h. Halleux et al. (1985) trabalhando em casa de vegetação com cobertura de vidro, verificaram que após a incidência máxima da radiação, as temperaturas continuam aumentando por consequencia do efeito do aquecimento do solo e outros corpos, que difundem calor aquecendo $o$ ar adjacente em cultivos protegidos.

A temperatura média interna do ambiente estufa $\left(28,8^{\circ}\right)$ foi maior que a temperatura externa $\left(23,9^{\circ}\right)$, durante o período experimental, sendo que o maior aquecimento do ar, sob as coberturas de plástico, foi responsável pelas maiores correlações existente entre as variáveis: RFA e temperatura da folha com a temperatura interna, comparado às correlações existentes entre as mesmas e a temperatura externa.

De acordo com Bohmer et al. (2008) o ambiente protegido, como é o caso das estufas, modifica o comportamento da temperatura do ar em seu interior comparado ao ambiente externo. O maior efeito em termos térmicos ocorre no aumento das temperaturas médias pelo aumento das temperaturas máximas. A ideia exposta por Zanella et al. (2006) afirma ocorrer interferência nas respostas das plantas aos processos fisiológicos, tais como a transpiração e a fotossíntese, em função dos diferentes materiais utilizados para a cobertura de ambientes.

Enquanto a resistência estomática aumentou, a transpiração apresentou comportamento inverso, uma vez que essas duas variáveis apresentaram correlação negativa altíssima entre si $(-0,96)$. Complementando a ideia proposta por Messinger et al. (2006) e Taiz \& Zeiger. (2009), pode-se inferir que a condutância e a resistência estomática são variáveis inversamente proporcionais.

De acordo com as correlações existentes entre as características fisiológicas e a temperatura, pode-se inferir que este é um fator ambiental de altíssima importância, pois sua variação pode modificar consideravelmente a fisiologia da planta, e, por consequência, comprometer sua taxa metabólica e seu crescimento.

De modo geral, à medida que a radiação solar aumenta, a temperatura da folha e a resistência estomática aumentam, ao passo que a transpiração diminui (Figura 1); dessa forma, pode-se verificar que no momento em que fatores abióticos modificam a temperatura foliar desta espécie, a sua atividade metabólica tende a oscilar de forma proporcional à magnitude dos fatores ambientais.

Este resultado reforça a ideia proposta por

TABELA 1. Coeficiente de correlação de Pearson existente entre as variáveis fisiológicas e elementos climáticos, onde TF: temperatura da folha; RFA: radiação fotossinteticamente ativa; RE: resistência estomática; TR: transpiração; TI: temperatura no interior da estufa; TE: temperatura no exterior da estufa.

\begin{tabular}{|c|c|c|c|c|c|}
\hline \multirow{2}{*}{ Atributo } & \multicolumn{5}{|c|}{ Coeficiente de correlação } \\
\hline & TF & RFA & RE & TR & TI \\
\hline RFA & $0,66^{* *}$ & & & & \\
\hline $\mathrm{RE}$ & $0,58^{\mathrm{ns}}$ & $-0,06^{\text {ns }}$ & & & \\
\hline TR & $-0,50^{\text {ns }}$ & $0,13^{\text {ns }}$ & $-0,96^{* *}$ & & \\
\hline $\mathrm{TI}$ & $0,91^{* *}$ & $0,87^{\star *}$ & $0,34^{\mathrm{ns}}$ & $-0,30^{\text {ns }}$ & \\
\hline TE & $0,70^{* *}$ & $0,02^{\mathrm{ns}}$ & $0,79^{* *}$ & $-0,81^{* *}$ & $0,43^{\text {ns }}$ \\
\hline
\end{tabular}

${ }^{\text {ns }}$ Não significativo em nível de $1 \%$ de probabilidade de erro pelo teste t de Student. ${ }^{* *}$ Significativo em nível de $1 \%$ de probabilidade de erro pelo teste $t$ de Student.

Rev. Bras. PI. Med., Campinas, v.16, n.2, p.196-201, 2014. 

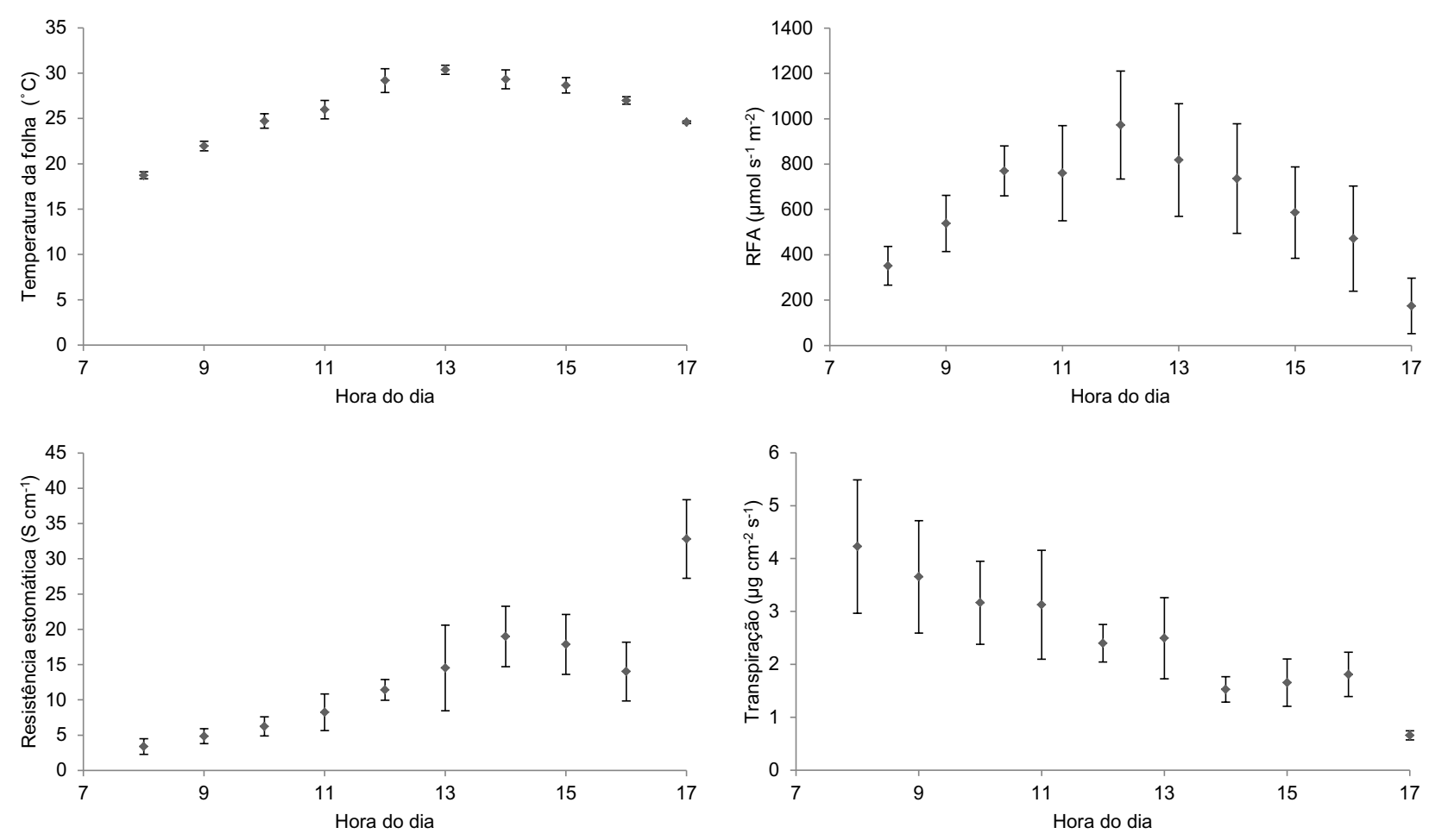

FIGURA 1. Valores da média e intervalo de confiança da radiação solar fotossinteticamente ativa (RFA), temperatura da folha, resistência estomática e transpiração, em condições atmosféricas de céu claro, no município de Frederico Westphalen, ao longo do dia 10/02/2011.

Casaroli et al. (2008) ao observar que a taxa de transpiração das folhas é diretamente influenciada pelo saldo de radiação, da condutância estomática, e do déficit de saturação de vapor no ar.

O fechamento estomático, ocorrido principalmente nas horas mais quentes do dia, prejudica a atividade fotossintética pelo fato de impedir a entrada de $\mathrm{CO}_{2 ;}$ portanto, acarreta redução do crescimento e do desenvolvimento da planta (Taiz \& Zieger, 2009).

A taxa de transpiração foi menor às 17:00h $\left(0,658 \mathrm{mmol} \mathrm{H}_{2} \mathrm{O} \mathrm{s}^{-1} \mathrm{~m}^{-2}\right)$, no momento em que a resistência estomática apresentou seu valor máximo $\left(32,8 \mathrm{~S} \mathrm{~cm}^{-1}\right)$. A diminuição da taxa transpiratória nem sempre representa o estresse hídrico, mas o fechamento estomático é a estratégia utilizada pelas plantas para manter a turgescência durante $o$ período em que ocorre maior demanda de vapor de água pela atmosfera. Se esta taxa propriamente dita reduzir a valores menores que um determinado valor crítico da espécie, poderá ocorrer o estresse hídrico, acarretando perdas de produtividade (Casaroli et al., 2008).

Resultados observados por Marin et al. (2003) fortalecem esta ideia, afirmando que a taxa de transpiração do cafeeiro esteve relacionada principalmente com a radiação solar e com o déficit de pressão de vapor no ar. Outro fator que está relacionado com a taxa de transpiração da planta é o potencial de água no substrato. Segundo Paiva et al. (2005) ao trabalharem com medições de porometria na cultura do feijoeiro, observaram relação alta e direta entre a condutância estomática e o potencial da água no solo.

De modo geral, os menores coeficientes de variação encontrados para os parâmetros estudados foram entre 10:00h e 13:00h (Figura 2); dessa forma, podemos inferir que neste intervalo de tempo as variáveis fisiológicas oscilam com menor intensidade, tornando a determinação mais eficiente.

Variações mais intensas dos componentes fisiológicos da planta podem comprometer a determinação do comportamento fisiológico da mesma devido a sua grande instabilidade. O equilíbrio dinâmico do sistema solo-plantaatmosfera é o responsável pela ocorrência de menores variações das características estudadas. Sendo assim, a determinação destas variáveis em horários inadequados pode acarretar uma elevada oscilação dos componentes fisiológicos da planta, comprometendo os resultados da avaliação. 

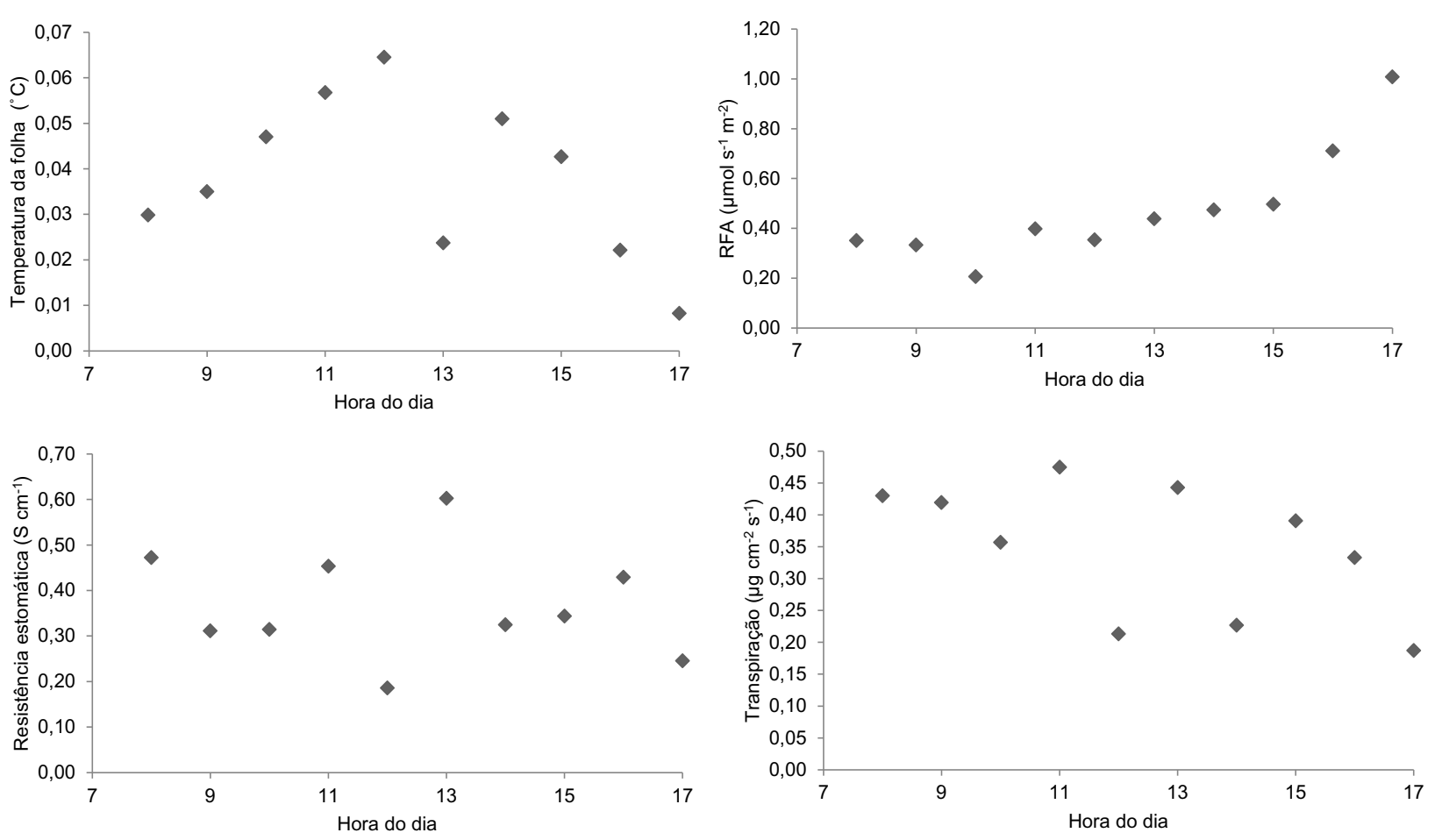

FIGURA 2. Coeficiente de variação encontrado para as variáveis: radiação solar fotossinteticamente ativa (RFA), temperatura da folha, resistência estomática e transpiração, em condições atmosféricas de céu claro, no município de Frederico Westphalen, ao longo do dia 10/02/2011.

\section{CONCLUSÃO}

A espécie Bauhinia forficata apresenta oscilações no seu mecanismo fisiológico em função do horário e das condições climáticas a qual esteja submetida.

O período diário mais indicado para a realização de avaliações, tais como: temperatura da folha, radiação fotossinteticamente ativa, resistência estomática e transpiração em plantas de Bauhinia forficata é das 10:00 às 13:00 horas.

\section{REFERÊNCIA}

AGRA. M.F. et al. Synopsis of the plants known as medicinal and poisonous in Northeast of Brazil. Revista Brasileira de Farmacognosia, v. 17, n.1, p. 114-140, 2007.

AMARAL, J.A.T. et al. Crescimento vegetativo sazonal do cafeeiro e suas relações com fotoperíodo, frutificação, resistência estomática e fotossíntese. Pesquisa Agropecuária Brasileira, v. 41, n. 3, p. 377-384, 2006.

BÖHMER, C.R.K. et al. Alterações na temperatura do ar proporcionadas por estufa de polietileno, durante um cultivo de feijão-vagem. Revista Brasileira de Agrometeorologia, v.16, n. 2, p.143-148, 2008.

CARVALHO, P.E.R. Espécies florestais brasileiras: recomendações silviculturais, potencialidades e uso da madeira. 1. ed. Brasília: EMBRAPA-SPI, 1994. $640 p$.

CASAROLI, D. et al. Estimativa da taxa de transpiração do feijoeiro a partir de medidas de porometria, em diferentes camadas do dossel. Revista Brasileira de Agrometeorologia, v.16, n. 2, p.149-153, 2008.

COSTA, G.F.; MARENCO, R.A. Fotossíntese, condutância estomática e potencial hídrico foliar em árvores jovens de andiroba (Carapa guianensis). Acta Amazonica, v. 37, n.2, p. 229-234, 2007.

FLOSS, E.L. Fisiologia das plantas cultivadas. Passo Fundo: UPF, 2004. 536p.

HALEUX, D. et al. Dynamic simulation of heat fluxes and temperatures in horticultural and low emissivity glasscovered greenhouses. Acta Horticulturae, v.170, p.91-6, 1985.

JADOSKI, S.O. et al. Relações hídricas e fisiológicas em plantas de pimentão ao longo de um dia. Ambiência, v. 1, n. 1, p. 11-19, 2005.

JONES, J.W. et al. Plant resistance to water flow in field soybeans: I. Non-limiting soil moisture. Agronomy journal, v.74, p.92-105, 1982.

LORENZI, H. Árvores brasileiras: manual de identificação e cultivo de plantas arbóreas nativas do Brasil. 2. ed. Nova Odessa: Plantarum, 2002, 368p.

MALUF, J.R.T. Nova classificação climática do Estado do Rio Grande do Sul. Revista Brasileira de Agrometeorologia, v. 8, n. 1, p. 141-150, 2000.

MARENCO, R.A.; LOPES, N.F. Fisiologia Vegetal: Fotossíntese, Respiração, Relações Hídricas e Nutrição Mineral. 1. ed. Viçosa: Editora UFV, 2005. $451 \mathrm{p}$.

MARIN, F.R. et al. Solar radiation interception and its relation with transpiration in different coffee canopy 
layers. Revista Brasileira de Agrometeorologia, v.11, n.1, p. 1-6, 2003.

MENEZES, F.S. et al. Hypoglycemic activity of two Brazilian Bauhinia species: Bauhinia forficata L. and Bauhinia monandra Kurz. Revista Brasileira de Farmacognosia, v.17, n.1, p. 8-13, 2007.

MESSINGER, S.M. et al. Evidence for involvement of photosynthetic processes in the stomatal response to $\mathrm{CO}_{2}$. Plant Physiology, v. 140, n. 2, p. 771-778, 2006.

PAIVA, A.S. et al. Condutância estomática em folhas de feijoeiro submetido a diferentes regimes de irrigação. Engenharia Agrícola, v.25, n.1, p.161-169, 2005.

PEIXOTO, P.H.P. et al. Responses of the photosynthetic apparatus to aluminum stress in two sorghum cultivars. Journal of Plant Nutrition, v. 25, n. 4, p. 821-832, 2002.

ROSA, D.D. et al. Ocorrência de Oídio (Oidium caesalpiniacearum Hosag \& W. Braum) em Pata de Vaca (Bauhinia forficata Link.) no Brasil. Summa Phytopathologica, v. 34, n. 2, p. 196, 2008.

SAS LEARNING EDITION. Getting started with the SAS Learning Edition. Cary, 2003. 200p.

SILVA, K.L. et al. Phytochemical and pharmacognostic investigation of Bauhinia forficata (Leguminosae). Zeitschrift Fur Naturforschung C - A Journal of Biosciences, v. 55, n. 5-6, p. 478-480, 2000.
SILVA, K.L.; CECHINEL FILHO, V. Plantas do gênero Bauhinia: Composição química e potencial farmacológico. Química Nova, v. 25, p. 449-454, 2002.

SOUZA, C.C. et al. Avaliação de métodos de determinação de água disponível e manejo de irrigação em terra roxa sob cultivo de algodoeiro herbáceo. Revista brasileira de engenharia agrícola e ambiental, v. 4, n. 3, p. 338-342. 2000.

SOUSA, E. et al. Hypoglycemic effect and antioxidant potential of kaempferol-3,7-O-( $\alpha$ )-dirhammnoside from Bauhinia forficata leaves. Journal of Natural Products, v. 67 , p. 829-832, 2004.

SUAREZ, S. et al. Aloysia citriodora: morphology and density of gladular trichomes, and its relationships with essential oil content. In: SIMPÓSIO BRASILEIRO DE ÓLEOS ESSENCIAIS, 2., 2003, Campinas. Anais... Campinas: Instituto Agronômico, 2003. p. 78.

STEEL, R.G.R.; TORRIE, J.H. Principles and procedures of statistics: A Biometrical Approach. 2. ed. London: McGraw-Hill, 1980. 666p.

TAIZ, L.; ZEIGER, E. Fisiologia vegetal. 3. ed. Porto Alegre: Artmed, 2009. 848p.

ZANELLA, F. et al. Formação de mudas de maracujazeiroamarelo com níveis de sombreamento em Ji-Paraná - RO. Ciência e Agrotecnologia, v.30, n.5, p.880-884, 2006.

Rev. Bras. PI. Med., Campinas, v.16, n.2, p.196-201, 2014. 